Präv Gesundheitsf 2021 · 16:157-162 https://doi.org/10.1007/s11553-020-00798-6 Eingegangen: 6 . April 2020

Angenommen: 6. Juni 2020

Online publiziert: 26. Juni 2020

(c) Der/die Autor(en) 2020

Monika Urban

Institut für Public Health und Pflegeforschung, Universität Bremen, Bremen, Deutschland

\title{
Geschlechtersensible Gestaltung digitaler Gesundheitsförderung
}

tuiert [10] und oftmals mit spezifischen Gesundheitsverhalten (Yogakurse oder „Gamification“ zur Gesundheitsförderung), täglichen gesundheitsbezogene Routinen (Rauchen oder Medikamenteneinnahme bei Stress) und typischen Belastungen (Feinstaublunge eines Lackierers oder Bandscheibenvorfälle einer Pflegefachkraft) korrespondiert [17]. Gesundheitsbedarfe und Krankheitsverläufe werden mit eben dieser langfristigen und dynamischen Materialisierung von Körpern (ergo den Auswirkungen von Veranlagungen, sich ändernden Ressourcen und Belastungen) erklärt [18], an die die Gesundheitsförderung mit möglichst passgenauen Interventionen ansetzt.

Das soziale Geschlecht wird bei geschlechtersensiblen Interventionen nicht isoliert betrachtet. Vielmehr wird es mit sozialen Markern wie Alter, sexuelle Orientierung, Behinderung, Ethnizität, kultureller Hintergrund sowie sozialer Schichtzugehörigkeit verknüpft, um Aussagen über die Verteilung spezifischer Gesundheitsverhalten und Erkrankungen machen zu können [1]. Ein solcher gendersensibler und diversitätsorientierter Ansatz der Gesundheitsförderung legt sich entsprechend ein komplexes Zusammenspiel zwischen sozialökonomischen und kulturell geprägten Belastungen und Ressourcen, verschiedenen Genderidentitäten und dem individuellen Organismus vor [3]. Flankiert wird dieser Ansatz durch eine Gesundheitspolitik, die ein Gender-Mainstreaming, also die Einbindung von Menschen verschiedener Gender und damit die Gewährleistung der gleichberechtigten Teilhabe an politischen, wirtschaftlichen und gesellschaftlichen Prozessen, sowie dafür im Konkreten auch die verbesserte Patient ${ }^{*}$ innen- sowie Nutzer*innenbeteiligung befördern will, um den Bedarfen möglichst umfangreicher Teile der Bevölkerung optimal gerecht zu werden [25].

Im Zuge der Etablierung digitaler Infrastrukturen und Angebote ergeben sich neue Optionen für Interventionen der Gesundheitsförderung - und aus diesen resultieren auch für gendersensible und diversitätsorientierte Perspektiven neue Herausforderungen. Anhand zweier Fragestellungen soll die bisher verfügbare Evidenz aufgearbeitet werden:

1. Inwiefern wirken sich die Gender auf Effekte digitaler Interventionen der Gesundheitsförderung aus?

2. Welche möglichen Bedarfe an gendersensiblen Ansätzen lassen sich daraus ableiten?

\section{Material und Methode}

Der Artikel basiert auf einem scoping review, das nationale und internationale Studien einschließt, die mittels Literaturrecherche identifiziert und selektiv zusammengefasst wurden. Die Recherche wurde im Mai 2020 in den Datenbanken PubMed, Web of Science und Google Scholar (deutsche Seiten) durchgeführt. Gesucht wurde mit den Schlagwörtern "gender“, „digital“ und „health promotion". Es ergaben sich insgesamt 238 Treffer. Neben 18 Duplikaten wurden zwei Artikel in Spanisch, vier Ankündigungen sowie 25 Konferenzbeiträge entfernt. Exkludiert wurden 167 weitere Artikel, da ihnen ein inhaltlicher Bezug zu den gestellten Fragen fehlte (•Tab. 1).

Die Studienlage zur Interferenz von Gendern zu den Effekten digitaler Angebote ist bisher schwach [9]. Kein Treffer setzt sich dezidiert mit den hier gestellund über eine lange Zeit hinweg konsti- 


\section{Übersicht}

Tab. 1 Darstellung der strukturierten Literatursuche

Identifikation In Datenbanken gefunden: Web of

Science $n=40$, PubMed $n=45$

Vorauswahl Nach Entfernung der Duplikate Exkludiert $n=167$ $n=195$

\begin{tabular}{l|l|l}
\hline Eignung & Eignung von Volltexten $n=28$ & Exkludiert $n=7$ \\
\hline Auswahl & Inkludiert $n=21$ & -
\end{tabular}

ten Fragen auseinander. Die inkludierten Studien verweisen jedoch exemplarisch mit Blick auf einzelne Interventionen, Technologien und Zielgruppen darauf, inwiefern diese

a) negative Effekte haben, da sie Gender nicht berücksichtigen $[2,5,7,11,15$, $16,20,23,24]$,

b) einen Einfluss auf die Manifestation von Genderstereotypen haben $[8,9$, 22] und

c) von einer gendersensiblen Perspektive profitieren $[4,5,14,26]$.

Die meisten Studien erfassen Gender als die Kategorie der Dichotomie Frau vs. Mann (Ausnahmen: [5, 7, 26]). Dargestellt werden die Ergebnisse im Folgenden als Beantwortung der ersten Frage und dies entlang der drei Themenfelder. Im nächsten Schritt wird die zweite Frage auf Grundlage der dargestellten Ergebnisse beantwortet. Der Artikel endet mit einem hieraus entwickelten Fazit für die Praxis.

\section{Negative Effekte durch fehlende Gendersensibilität}

Digitale Interventionen haben die Gesundheitsförderung und Prävention multipliziert und diversifiziert: Eine kaum überschaubare Zahl von OnlinePlattformen, Foren und Apps informieren über Erkrankungen und Kampagnen, regen zu präventivem Verhalten an, messen medizinische Werte und begleiten therapeutische Interventionen. Gesundheitswissenschaftliche Studien zeigen, dass Apps moderat positive Effekte in Bezug auf Gesundheitskompetenz und Verbesserung der Gesundheitsversorgung haben, selbst im Vergleich zu entsprechenden konventionellen Interventionsmaßnahmen [20]. Bislang gilt allerdings als zentrales Manko, dass verbindliche Qualitätssiegel für Apps fehlen. Interventionen können insofern unpassende oder fehlerhafte Materialien (z.B. zugrunde liegende Studien oder Annahmen über die Zielgruppe) einbeziehen, woraufhin auf diesen basierende Algorithmen irreführende bis falsche Empfehlungen oder Gesundheitsprognosen generieren können [24].

Techniksoziologische Studien zeigen erstens, inwiefern dies für Apps zutrifft, die explizit für Bedarfe von menstruierenden Frauen entwickelt sind: Manche Zyklus-Apps kranken an Algorithmen, die auf wissenschaftlich überholten Methoden wie der Kalendermethode (Knaus-Ogino) basieren. Diese genoss ihre Hochphase in der Gynäkologie der 1950er-Jahre und dechiffriert die (vermeintlich) fruchtbaren Tage entlang eines fixen Rhythmus [11]. Aufgrund gynäkologischer Expertise ist diese Methode verworfen worden, da erwiesen ist, dass Zyklen von Stress, Ernährung und weiteren Faktoren abhängen. Dennoch suggeriert gerade die Visualisierung durch eine App, es handele sich um eine tatsächliche Aussage über den individuellen Körper [2].

Zweitens verweisen Studien darauf, dass irreführende bis falsche Empfehlungen oder Gesundheitsprognosen nicht alle Gender gleichermaßen treffen: Frauen und Inter- und Transsexuelle waren in der medizinischen und gesundheitswissenschaftlichen Forschung lange Zeit unterrepräsentiert. Eklatante Auswirkungen hatte dies aufSchlussfolgerungen basierend auf Studien zu Herz-KreislaufErkrankungen, da diese lange Zeit als „male-only studies“ durchgeführt und als geschlechtsneutral und allgemeingültig präsentiert wurden. Im Kontrast hierzu zeigen aktuelle differenzierende Studien, dass geschlechtsspezifische Unterschiede in jedem Stadium von Risikofaktoren, Entwicklung, Symptomatik und sogar in Bezug auf Überlebenschancen, Schutz- faktoren sowie Rehabilitation bestehen. Frauen und Inter- und Transsexuelle können also nur auf der Grundlage differenzierter Studien eine evidenzbasierte Therapie erwarten [18].

Solange Algorithmen aber ein (zumeist) unüberprüfbares Betriebsgeheimnis darstellen und es damit auch unklar bleibt, ob geschlechtsspezifische Unterschiede ausreichend berücksichtigt sind, mangelt es potenziell an reliablen und validen Diagnosen. So können in digitalen (wie analogen) Angeboten zur Symptomerkennung veraltete Datengrundlage dazu führen (Frauen und Inter- und Transsexuelle zeigen bspw. weniger häufig die anerkannten Symptome eines Myokardinfarkts), dass Hilfebedarf zu spät angezeigt werden [23].

Drittens zeigen sich negative Effekte sogar insofern, als dass sich gesundheitsförderliche Potenziale ins Gegenteil verkehren können, wenn gesellschaftliche Stigmatisierungen von Gendern nicht bedacht werden. Evident wird dies bei Angeboten, wie Online-Foren und Gesundheitsplattformen, die zu Verhaltensänderungen anregen und Gesundheitsinformationen verbreiten. Diese bieten häufig die Funktion an, benutzergenerierte Beiträge hochzuladen und jene anderer User zu kommentieren. Ein solch interaktives Verfahren birgt - neben vielen Vorteilen - Gefahren: Eine inhaltsanalytische Studie von Kommentaren zu Videos von Personen auf einem Forum für übergewichtige Menschen arbeitet heraus, dass negative Kommentare die unterstützenden überwogen. Im Besonderen erhielten Videos von Frauen herabwürdigende Anmerkungen und dies überproportional häufig durch User, die sich männlich definierten [16].

Auch eine Studie über Angebote auf Social-Media-Plattformen für LGBTIQ (lesbisch, schwul, bisexuell, trans- und intergender und -sexuell, queer) Jugendliche kommt zu einem ähnlichen Ergebnis. Die Jugendlichen gehören bei ihrer sexuellen Identitätsbildung einer sexuellen Minorität an und passende Unterstützungsangebote können zur Gesundheitsförderung beitragen, indem sie fehlende Rollenmodelle und Ansprechpartner*innen stiften und zumeist auch dabei helfen, Diskriminierungserfahrungen zu 


\section{Urban}

\section{Geschlechtersensible Gestaltung digitaler Gesundheitsförderung}

\section{Zusammenfassung}

Hintergrund. Die Kategorie Geschlecht hat für die Gesundheit eine zentrale Bedeutung und geschlechtersensible Perspektiven gehören entsprechend zum wissenschaftlichen Standard der Gesundheitsförderung und Prävention. Durch digitale Interventionen der Gesundheitsförderung ergeben sich nun neue Herausforderungen, da ein Einfluss der Kategorie Gender auf die Wirkung solcher Interventionen zwar prinzipiell bekannt ist, die konkreten Effekte fehlender gendersensibler Perspektiven bisher jedoch nicht systematisiert sind.

Ziel der Arbeit. Der Artikel stellt potenzielle unintendierte Effekte durch fehlende gendersensible Perspektiven vor und diskutiert Maßnahmen einer gender- und diversitätssensiblen Gestaltung digitaler Interventionen der Gesundheitsförderung. Material und Methode. Der Artikel basiert auf einem scoping review, das nationale und internationale Studien einschließt.

Ergebnisse. Die Studienlage zur Interferenz von Gendern zu den Effekten digitaler Angebote ist bisher schwach. Die inkludierten Studien verweisen exemplarisch mit Blick auf einzelne Interventionen, Technologien und Zielgruppen darauf, dass fehlende gendersensible Perspektiven negative Effekte haben. Außerdem zeigt sich, dass digitale Interventionen die Dichotomien entlang der Kategorien Frau vs. Mann (re)manifestieren können. Andere Körper, sowie ein anderes als heterosexuelles Begehren, können bei digitalen Interventionen unberücksichtigt bleiben.

Diskussion. Für eine Vermeidung unintendierter Effekte digitaler Interventionen bietet sich die Einbeziehung gendersensibler und diversitätsorientierter Perspektiven an. Ein durchgängig gendersensibles Design, Gendersensibilität als wissenschaftlicher Standard und partizipative, User-orientierte Technikentwicklung können unintendierte Effekte minimieren.

\section{Schlüsselwörter}

Digitalisierung · Gender · Digitale Interventionen · Diversität · Gendersensibles Design

\section{Gender-sensitive design of digital health promotion}

\section{Abstract}

Background. The category of gender is of high importance for health promotion. Thus, gender-sensitive research approaches are part of the scientific standard for health promotion and prevention programs. A new challenge emerges due to the range of new digital interventions because-although we know about the association between digital practices and gender-based disadvantage - little experience is available on gender-sensitive design for digital health technologies. Objectives. The article presents potential gateways for gender-specific disadvantages through digital interventions. Based on that, the review highlights the relevance of gender- sensitive designs, which could help reduce disadvantages and health equalities. Materials and methods. The article is based on a scoping review which includes national and international studies.

Results. The state of studies regarding the impact of gender on the effects of digital interventions is so far quite weak. The studies quoted in this scoping review point out, with regard to specific interventions, technologies and target groups, that a lack of gendersensitive perspectives has negative effects and can undermine positive effects of health interventions. It also shows that digital interventions can manifest and solidify the dichotomies along the categories of women versus men. Thereby, other gender identities, as well as desires other than heterosexuality, can be disregarded.

Conclusions. In order to avoid unintended effects, it is advisable to include gendersensitive and diversity-oriented perspectives. A consistent gender-sensitive design, gender sensitivity as a scientific standard and participatory, user-oriented design can minimize unintended effects.

\section{Keywords}

Digital health - Gender - Digital health promotion - Diversity - Gender-sensitive design verarbeiten. Allerdings sind diese Angebote auf der Seite der Inhaltsproduktion meistens männlich geprägt und nur für einen Teil der Jugendlichen attraktiv. Eine inhaltsanalytische Studie, die sich explizit jenen selteneren Angeboten für Frauen und Transpersonen zuwendet, verweist darauf, dass oftmals auf lesbische Selbstoffenbarungen auch ablehnende Reaktionen des Publikums folgen [7].

Viertens bedingt der sog. ,digitale divide“, also der ungleich verteilte Zugang zum Internet und der hieraus resultieren- de gewinnbringende Nutzen, dass nicht jede Person gleichermaßen von digitalen Angeboten profitieren kann. Neben sozialen und altersbedingten Einschränkungen bestehen globale, die sich nicht nur zwischen Ländern, zwischen ländlichem und städtischem Raum, zwischen Armutsvierteln und besser situierten Quartieren bemerkbar machen, sondern Frauen weltweit haben einen geringeren Zugang zu Online-Gesundheitsangeboten [12]. In vielen Ländern sind Frauen auf männliche Familienmitglieder angewiesen, um Angebote nutzen zu können, wodurch sie oftmals ihre Privatsphäre, Autonomie und Entscheidungsfreiheit einbüßen. Auf sie abzielende digitale Interventionen kranken wiederum daran, dass ihnen nur eingeschränkte Mitbestimmungsrechte zugestanden werden und somit die Angebote schwer auf ihre Bedürfnisse abzustimmen sind [13]. Ein solcher "digital gender gap“ kann gesundheitliche Ungleichheiten potenzieren [12]. Gleichzeitig zeigt ein systematisches „literature review“ über digitale Interventionen für Frauen, dass, werden diese entsprechend gendersensi- 
ble und soziale eingebettet angeboten, sie gesundheitsförderliche Entscheidungen begünstigen und den Zugang zur Gesundheitsversorgung verbessern können [15].

\section{Einfluss auf die Manifestation von Genderstereotypen}

Die meisten digitalen Technologien für die Gesundheitsförderung sind für alle Gender designt. Dennoch, so zeigen qualitative Studien, unterscheiden sich die Nutzungsverhalten auch entlang der Gender. Qualitative Studien heben hervor, dass Frauen häufiger Selftracking-Apps einsetzen, um ihren Kalorienverbrauch nachzuvollziehen sowie Trainingsprogramme nutzen, um Körperpartien zu formen, während Männer im Durchschnitt häufiger ihre körperliche Leistungsfähigkeit aufzeichnen und teilen. Auch wenn Nutzerinnen gemäß ihrer Interessen die Programme wählen, werden sie durch die in den Apps vorformatierten Ziele (sowie entlang von Bildgebungsverfahren und Praktiken) auf stereotypische traditionelle Körperideale ausgerichtet. Damit (re)manifestieren und verfestigen sich Dichotomien entlang der Kategorien Frau vs. Mann [22]: Einerseits aktualisieren sich hierdurch Genderhierarchien zwischen makellosen zierlichen, begehrlichen Frauen und leistungsstarken, begehrenden Männern [8]. Andererseits werden bestimmte Körper, in einer Studie expliziert an nichtweißen sowie trans- und intersexuellen Körpern, sowie von heterosexuellem Begehren abweichende Formen der Sexualität, als Abweichungen bestimmbar [8].

Eine andere qualitative Studie über Erfahrungen von Nutzerinnen verschiedener Fitness-Apps hebt außerdem hervor, dass die Reflexion über genderspezifisches Rollenverhalten immens wichtig ist, um unintendierte Effekte digitaler Fitnesstechnologien verstehen zu können. US-amerikanische Fitbit-Userinnen, die ihre gesundheitlichen Belastungen, Trainingsprogramme und Fitnessziele in entsprechenden Foren darlegten, monierten, wie die Studie nachvollzieht, dass sie die standardisierten Trainingsziele des Anbieters selbst un- ter größten Anstrengungen nicht erreichen konnten. Geschuldet war dies einer räumlichen und zeitlichen Belastung, ausgelöst durch gleichzeitig an sie gestellte Anforderungen des Arbeitsmarktes sowie durch reproduktive häusliche und familiäre Verpflichtungen. Im Vergleich beklagten männliche Nutzer solche Effekte nicht. Die Studie schloss entsprechend damit, dass die Wirkung von Apps nur unter Einbeziehung sozioökonomischer Faktoren und bei genderdifferenzierter Analyse nachzuvollziehen ist [9].

\section{Gendersensible Gesundheits- förderung mit Apps}

Einige Online-Interventionen basieren bereits auf gendersensiblen Ansätzen. Diese sind jedoch nicht per se Erfolg versprechend. Demgemäß arbeitet eine Studie über Online-Gesundheitsinformationen heraus, dass Frauen häufiger als Männer Online-Gesundheitsinformationen suchten, dass aber ein gewinnbringender Nutzen von sozioökonomischen Faktoren abhing [4]. In diesem Sinne zeichnet eine andere Studie über die visuelle und sprachliche Gestaltung einer kanadischen Gesundheitskampagne zur Prävention von Herz-Kreislauf-Erkrankungen bei Frauen nach, dass ein nichtinklusives Design einer online Kampagne schaden kann: Diese versäumte nämlich ethnische, sexuelle und anderer Minderheiten in den Materialien zu repräsentieren, woraufhin Personengruppen dieses Angebot als nicht für sich relevant wahrnahmen. Die Intervention erwies sich dadurch als nur begrenzt wirksam [5].

Von einem gelingenden gendersensiblen Ansatz berichtet wiederum ein anderer Projektbericht. Im Rahmen der Entwicklung von digitalen Unterstützungsangeboten zum Diabetes-Selbstmanagement entwickelte das Projekt ein Modell, mit dem das Selbstmanagement entlang von differenzierten Verhaltenstipps verbessert werden könne. Dabei basierten die Tipps auf Studien zu biologischen, verhaltensbezogenen und psychosozialen Faktoren. Die im Projekt entwickelten Unterstützungsangebote wurden jedoch anlassbezogen und nicht entlang der Angaben zum Gender generiert. So erhielten auch Männer Empfehlungen zum Anlass „geringe Selbstwirksamkeit“, obwohl die Studiengrundlagen dies als ein Spezifikum der durchschnittlichen Krankheitserscheinungen von Frauen auswiesen. Ebenso wurden auch keine automatisierten Tipps an Frauen zur Vermeidung schlechter Gemütszustände versandt, obwohl Daten zeigen, dass Frauen durchschnittlich häufiger von depressiven Verstimmungen betroffen sind. Neben den anlassbezogenen Gesundheitstipps konnten Nutzer*innen auch ihre eigenen Spezifizierungen vornehmen, bspw. in Form von persönlichen Präferenzen: Als Beispiel wurde zitiert, dass Nutzer*innen Grenzwerte ihres Alkoholkonsums festlegen konnten, die mit ihren Gewohnheiten und Verträglichkeiten korrespondierten. Es war Teil der diversitätsorientierten und gendersensiblen Herangehensweise, diese nicht entlang der in der Literatur festgelegten geschlechtsspezifischen Grenzwerte automatisiert festzulegen [26].

Resümierend lässt sich die Frage nach den Auswirkungen von Gendern auf die Effekte der digitalen Interventionen insofern beantworten, als dass die Interferenzen facettenreich sind: Es bedarf in der Technologieentwicklung einer Einbeziehung gendersensibler Perspektiven, um unintendierte Effekte zu vermeiden. Gleichzeitig müssen diese kontextabhängig mit diversitätsorientierten Ansätzen verschränkt sein, um neue Vulnerabilitäten durch die digitalen Interaktionen zu minimieren.

\section{Bedarfe an gendersensiblen Perspektiven}

Die Studien des Reviews verweisen darauf, dass gendersensible Ansätze für digitale Interventionen relevant sind, um unintendierte negative Effekte $\mathrm{zu}$ reduzieren und die Vertiefung struktureller Ungleichheiten zu vermeiden. Zur Realisierung erfolgreicher digitaler Interventionen sind in der Entwicklung jedoch nicht nur gendersensible Ansätze zu wählen, sondern diese mit weiteren Kategorien wie genderspezifische Diskriminierung, sexuelle Orientierung, sozioökonomischer Status und kultureller Hin- 
tergrund zu verschränken, damit digitale Interventionen angenommen werden (können; [21]).

Eine Reaktion hierauf ist die Entwicklung von diversitätsorientierter und/oder barrierefreier Soft- und Hardware, die unterschiedlichen Vorlieben und Bedarfen sowie körperlichen, sprachlichen, technischen und kognitiven Kompetenzen gerecht wird. Ein solches Design stellt die entwickelnden Personen - mehrheitlich junge, männliche eTechniker, Informatiker und Produktdesigner mit höherem Bildungsgrad sind - vor eine Herausforderung [26]: Die von ihnen entwickelten Technologien spiegeln ihre Vorstellungen über die Wünsche, Kompetenzen und Bedarfe ihrer anvisierten potenziellen Nutzer*innen wieder. Die Diskrepanz zwischen vermuteten und tatsächlichen Kompetenzen und Bedarfen kann dazu beitragen, dass Angebote potenzielle Zielgruppen verfehlen [14]. Gegengesteuert werden kann dadurch, dass im Forschungs- und Entwicklungsteam Gender- und Diversitätskompetenzen einbezogen werden [26].

Auf der Versorgungsebene können politische Rahmenbedingungen bspw. zur verbindlichen Regelung von Kassenübernahmen, wie jüngst partiell über das Digitale-Versorgung-Gesetz realisiert, den Zugang zu Angeboten verbessern. Hierin besteht eine große Chance, denn Studien zeigen, dass online Gesundheitsangebote im Besonderen für sozial benachteiligte Frauen gesundheitsförderlich wirken können [19].

Resümierend lässt sich folgern, dass bereits vorliegende Erfahrungen aus (analogen) Interventionen nicht unbedingt ausreichen, um unintendierte Effekte zu vermeiden: Algorithmen, digitalen Bildgebungsverfahren und OnlineKommunikationen wohnen neue Risiken inne. Offene Gesundheitsforen oder Gesundheitsangebote auf Social Media bedürfen so beispielsweise einer zeitnahen Redaktion, denn die Anonymität digitaler Kommunikation begünstigt die Zunahme genderspezifischer (digitaler) Gewalt, die häufig eine Ergänzung oder Verstärkung von bereits offline erfahrenen Gewaltdynamiken darstellt.

\section{Fazit für die Praxis}

- Um mit qualitativ hochwertigen Interventionen große Teile der Bevölkerung zu erreichen und gesundheitlicher Ungleichheit entgegen zu treten, bedarf es Gendersensibilität als Kernkompetenz. Allerdings wird es für eine gendersensible Gestaltung der Digitalisierung keine einfache Checkliste oder Rezeptur geben. Es bedarf vielmehr langfristiger Lernprozesse und Reflektionen von Technikentwicklung, Implementierungen und Techniknutzung in actu.

- Hierfür bieten die Kernelemente der gendersensiblen Ansätze der Gesundheitsförderung, wie eingangs ausdifferenziert, eine Basis: Dies würde erstens eine Sicherstellung des Gender-Mainstreaming-Prinzips in der Technikentwicklung bedeuten, das ein durchgängig gendersensibles Design befördert. Zweitens wäre die Verschränkung von Gender- und Diversitätssensibilität als Kriterium guter Interventionen festzuhalten. Drittens befördert partizipative User-orientierte Technikentwicklung Interventionen, die heterogenen Bedarfen verschiedener Gender, Bedarfe und Bevölkerungsgruppen entsprechen.

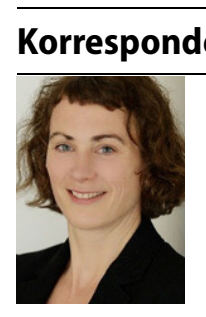

Institut für Public Health und Pflegeforschung, Universität Bremen

Grazer Str. 2, 28359 Bremen, Deutschland murban@uni-bremen.de

Funding. Open Access funding provided by Projekt DEAL.

\section{Einhaltung ethischer Richtlinien}

Interessenkonflikt. M. Urban gibt an, dass kein Interessenkonflikt besteht.

Für diesen Beitrag wurden von der Autorin keine Studien an Menschen oder Tieren durchgeführt. Für die aufgeführten Studien gelten die jeweils dort angegebenen ethischen Richtlinien.
Open Access Dieser Artikel wird unter der Creative Commons Namensnennung 4.0 International Lizenz veröffentlicht, welche die Nutzung, Vervielfältigung Bearbeitung, Verbreitung und Wiedergabe in jeglichem Medium und Format erlaubt, sofern Sie den/die ursprünglichen Autor(en) und die Quelle ordnungsgemäß nennen, einen Link zur Creative Commons Lizenz beifügen und angeben, ob Änderungen vorgenommen wurden.

Die in diesem Artikel enthaltenen Bilder und sonstiges Drittmaterial unterliegen ebenfalls der genannten Creative Commons Lizenz, sofern sich aus der Abbildungslegende nichts anderes ergibt. Sofern das betreffende Material nicht unter der genannten Creative Commons Lizenz steht und die betreffende Handlung nicht nach gesetzlichen Vorschriften erlaubt ist, ist für die oben aufgeführten Weiterverwendungen des Materials die Einwilligung des jeweiligen Rechteinhabers einzuholen.

Weitere Details zur Lizenz entnehmen Sie bitte der Lizenzinformation auf http://creativecommons.org/ licenses/by/4.0/deed.de.

\section{Literatur}

1. Altgeld T, Kolip P (2006) Geschlechtergerechte Gesundheitsförderung und Prävention: Ein Beitrag zur Qualitätsverbesserung im Gesundheitswesen. In: Kolip P, Altgeld T (Hrsg) Geschlechtergerechte Gesundheitsförderung und Prävention. Juventa, Weinheim, S15-26

2. Amelang K (2019) Monatliche Blutflüsse als Gesprächsstoff? Zur Neuverhandlung der Menstruation in digitalisierten Zeiten. Schweiz Arch Volkskd 115(1):65-80

3. Babitsch B (2006) Die Kategorie Geschlecht: Implikationen für den Zusammenhang zwischen sozialer Ungleichheit und Gesundheit. In: Richter M, Hurrelmann K (Hrsg) Gesundheitliche Ungleichheit. Grundlagen, Probleme, Perspektiven. VS, Wiesbaden, S271-287

4. Chen W, Lee KH (2014) More than a search? Informational and participatory eHealth behaviors. Comput Human Behav 20:103-109

5. Clark Ml et al (2018) Taking a hard look at the Heart Truth campaign in Canada: a discourse analysis. J Health Psychol 23:1699-1710. https://doi.org/ $10.1177 / 1359105316669581$

6. Datta A, Tschantz M, Datta A (2015) Automated experiments on ad privacy settings. Proc Priv Enhanc Technol 1:92-112. https://doi.org/10. 1515/popets-2015-0007

7. Döring N, Prinzellner Y (2016) Gesundheitskommunikation auf YouTube: Der LGBTIQ-Kanal "The Nosy Rosie". In: Camerini AL, Ludolph R, Rothenfluh F (Hrsg) Gesundheitskommunikation im Spannungsfeld zwischen Theorie und Praxis, $S$ 248-259

8. Doshi MJ (2018) Barbies, goddesses, and entrepreneurs: discourses of gendered digital embodiment in women's health apps. Women's Stud Commun 41(2):183-203. https://doi.org/10.1080/ 07491409.2018 .1463930

9. Esmonde K, Jette S (2020) Assembling the ,Fitbit subject': a Foucauldian-sociomaterialist examination of social class, gender and selfsurveillance on Fitbit community message boards. Health 24(3):299-314

10. Ettore E, Kingdon C (2010) Reproductive regimes: governing gendered bodies. In: Kuhlmann E, 
Annandale E (Hrsg) The Palgrave handbook of gender and healthcare. Palgrave Macmillan, Basingstoke, S142-157 (hier 143)

11. Frank-Herrmann $P$, Freis $A$, Freundl-Schütt $T$ (2019) Zyklus-Apps zur Verhütung - sicher oder Gesellschaftsspiel? Gynäkologe 52:90-97. https:// doi.org/10.1007/s00129-018-4358-6

12. Garcia D et al (2018) Analyzing gender inequality through large-scale Facebook advertising data. Proc Natl Acad Sci U S A 115(27):6958-6963. https://doi.org/10.1073/pnas.1717781115

13. George AS et al (2018) Gender dynamics in digital health: overcoming blind spots and biases to seize opportunities and responsibilities for transformative health systems. J Public Health 40(2):ii6-ii11. https://doi.org/10.1093/pubmed/ fdy 180

14. Henwood F, Wyatt S (2019) Technology and in/ equality, questioning the information society. Digit Cult Soc 5(1):183-194 (hier 189)

15. Jennings L, Gagliardi L (2013) Influence of mHealth interventions on gender relations in developing countries: a systematic literature review. Int J Equity Health 12:85. https://doi.org/10.1186/ 1475-9276-12-85

16. Jeon YA et al (2018) Weight stigma goes viral on the internet: systematic assessment of youtube comments attacking overweight men and women. Interact J Med Res 7(1):e6. https://doi.org/10. 2196/ijmr.9182

17. Kolip P, Koppelin F (2002) Geschlechtsspezifische Inanspruchnahme von Prävention und Krankheitsfrüherkennung. In: Hurrelmann K, Kolip P (Hrsg) Geschlecht, Gesundheit und Krankheit. Männer und Frauen im Vergleich. Huber, Bern, S491-504

18. Kuhlmann E, Kolip P (2008) Die "gemachten" Unterschiede - Geschlecht als Dimension gesundheitlicher Ungleichheit. In: Bauer U, Bittlingmayer U, Richter M (Hrsg) Heath Inequalities. Determinanten und Mechanismen gesundheitlicher Ungleichheit. VS, Wiesbaden, S191-219

19. Lanpher MG etal (2016) Health literacy and weight change in a digital health intervention for women: a randomized controlled trial in primary care practice. J Health Commun 21:34-42. https://doi. org/10.1080/10810730.2015.1131773

20. Marcolino MS et al (2018) The impact of mHealth interventions: systematic review of systematic reviews. JMIR Mhealth Uhealth 6(1):e23. https:// doi.org/10.2196/mhealth.8873

21. Robinson $L$ et al (2025) Digital inequalities and why they matter. Inf Commun Soc 18(5):569-582. https://doi.org/10.1080/1369118X.2015.1012532 (hier 569)

22. Sanders R (2017) Self-tracking in the digital era: biopower, patriarchy, and the new biometric body projects. Body Soc 23(1):36-63

23. Schenck-Gustafsson K (2012) Coronary heart disease. In: Schenck-Gustafsson K et al (Hrsg) Handbook of clinical gender medicine. Karger, Basel, S 190-205

24. Schüz B, Urban M (2020) Unerwünschte Effekte digitaler Gesundheitstechnologien: Eine Public-Health-Perspektive. Bundesgesundheitsblatt Gesundheitsforschung Gesundheitsschutz 63:192-198. https://doi.org/10.1007/ s00103-019-03088-5

25. WHO Euro (2001) Mainstreaming gender equity in health. Madrid Statement. http://www.euro.who.
int/_data/assets/pdf_file/0008/76508/A75328. pdf.Zugegriffen: 31. März 2020

26. Zauchner-Studnicka S, Hornung-Prähauser V Plößnig M, Leutner M, Kautzky-Willer A (2016) Wie Gender in die Diabetes-SelbstmanagementApplikation kommt - ein vielversprechender Weg. GENDER 8(3):130-147 